\title{
Laplace Decomposition Method for the System of Non Linear PDEs
}

\author{
S. S. Handibag \\ Department of Mathematics, Mahatma Basweshwar Mahavidyalaya, Latur, Maharashtra, India \\ Email: sujitmaths@gmail.com
}

How to cite this paper: Handibag, S.S. (2019) Laplace Decomposition Method for the System of Non Linear PDEs. Open Access Library Journal, 6: e5954.

https://doi.org/10.4236/oalib.1105954

Received: November 25, 2019

Accepted: December 23, 2019

Published: December 26, 2019

Copyright $\odot 2019$ by author(s) and Open Access Library Inc.

This work is licensed under the Creative Commons Attribution International License (CC BY 4.0).

http://creativecommons.org/licenses/by/4.0/

\begin{abstract}
The Laplace Decomposition Method [1] [2] [3] is applied to a system of nonlinear partial differential equations to demonstrate potential applicability to such systems.
\end{abstract}

\section{Subject Areas}

Mathematical Analysis

\section{Keywords}

Laplace Decomposition Method, Partial Differential Equations, Laplace Transform

\section{Introduction}

Differential equations theory is an important Mathematical branch which is used to describe practical problems in physics, chemistry, and biology and so on [4]. It is well known that many phenomena in scientific fields such as reactiondiffusion process, population growth, solid physics, fluid dynamics, Mathematical biology and chemical kinetics, can be modelled by systems of linear or nonlinear PDEs. In order to understand and analyze these phenomena well, it needs to know solution of systems of these linear or nonlinear PDEs. So, it is a crucial work to obtain solutions of systems of linear or nonlinear PDEs in the science. With this idea; scientists and mathematicians have developed and searched some methods such as Hirota bilinear method [5], Exp-function method [6], tanh method [7] [8], sine-cosine method [9], Galerkin method [10] and Differential transform method (DTM) [11]. It is more difficult to obtain solutions of nonlinear PDEs than those of linear differential equations. Therefore, it may not always be possible to obtain analytical solutions of these equations [12] [13]. In 
this case, it is used analytical methods giving series solutions. In these kinds of methods, the solutions are sought in the form of series [1] [2] [3] [14]. Analytical methods are based on finding the other terms of the series from given initial conditions for the problem being considered. At this point, it is encountered the concept of convergence of the series. So, it is necessary to perform convergence analysis of these methods. As this convergence analysis can be carried out theoretically, one can gain information about the convergence of the series solution by looking at the absolute error between the numerical solution and the analytical solution. In some Analytic methods, a very good convergence can be achieved with only a few terms of the series, but more terms can be needed in some problems. That is, if the terms of the series increase, this provides better convergence to the analytical solution.

In this study, I have used LDM to solve a system of nonlinear partial differential equations for two different initial conditions. Later, we compared the obtained results with exact solutions and solution obtained by Method of Differential Quadrature [15]. In this paper, we are not going to explain the LDM. For that, I have referred papers [1] [2] [3] to illustrate this method for a nonlinear system of PDE's.

\section{Application}

Consider a system of nonlinear partial differential equations on our interest of region given by:

$$
\begin{gathered}
u_{t}=u u_{x}+v u_{y} \\
v_{t}=u v_{x}+v v_{y}
\end{gathered}
$$

with initial condition

$$
\begin{gathered}
u(x, y, 0)=f(x, y) \\
v(x, y, 0)=g(x, y)
\end{gathered}
$$

here, we have consider the general form of boundary conditions. Taking Laplace transform of Equations (2.1) and (2.2) with respect to $t$, we get

$$
\begin{gathered}
L_{t}\left[u_{t}\right]=L_{t}\left[u u_{x}+v u_{y}\right] \\
L_{t}\left[v_{t}\right]=L_{t}\left[u v_{x}+v v_{y}\right] \\
s u(x, y, s)-u(x, y, 0)=L_{t}\left[u u_{x}+v u_{y}\right] \\
s v(x, y, s)-v(x, y, 0)=L_{t}\left[u v_{x}+v v_{y}\right] \\
s u(x, y, s)=\frac{1}{s} f(x, y)+\frac{1}{s} L_{t}\left[u u_{x}+v u_{y}\right] \\
s v(x, y, s)=\frac{1}{s} g(x, y)+\frac{1}{s} L_{t}\left[u v_{x}+v v_{y}\right]
\end{gathered}
$$

Taking inverse Laplace transform of above system with respect to " $t$ ", we get

$$
u(x, y, t)=f(x, y)+L_{t}^{-1}\left[\frac{1}{s} L_{t}\left[u u_{x}+v u_{y}\right]\right]
$$




$$
v(x, y, t)=g(x, y)+L_{t}^{-1}\left[\frac{1}{s} L_{t}\left[u v_{x}+v v_{y}\right]\right]
$$

Let us suppose that,

$$
u(x, y, t)=\sum_{n=0}^{\infty} u_{n}(x, y, t), \quad v(x, y, t)=\sum_{n=0}^{\infty} v_{n}(x, y, t)
$$

be the solution of given system of Equations (2.1), (2.2) in series form. Also we can decompose the nonlinear terms appeared in given system by using adomian polynomials, namely

$$
u u_{x}=\sum_{n=0}^{\infty} A_{n}, v u_{y}=\sum_{n=0}^{\infty} B_{n}, u v_{x}=\sum_{n=0}^{\infty} C_{n}, v v_{y}=\sum_{n=0}^{\infty} D_{n}
$$

where $A_{n}, B_{n}, C_{n}$ and $D_{n}$ are adomian polynomials [16]. From the Equations (2.5), (2.6), (2.7) and (2.8), we get

$$
\begin{aligned}
& \sum_{n=0}^{\infty} u_{n}(x, y, t)=f(x, y)+L_{t}^{-1}\left[\frac{1}{s} L_{t}\left[\sum_{n=0}^{\infty} A_{n}+\sum_{n=0}^{\infty} B_{n}\right]\right] \\
& \sum_{n=0}^{\infty} v_{n}(x, y, t)=g(x, y)+L_{t}^{-1}\left[\frac{1}{s} L_{t}\left[\sum_{n=0}^{\infty} C_{n}+\sum_{n=0}^{\infty} D_{n}\right]\right]
\end{aligned}
$$

Comparing the both sides of above system of equations, we get the following recursive relations

$$
\begin{aligned}
& u_{0}(x, y, t)=f(x, y), u_{n+1}(x, y, t)=L_{t}^{-1}\left[\frac{1}{s} L_{t}\left[\sum_{n=0}^{\infty} A_{n}+\sum_{n=0}^{\infty} B_{n}\right]\right], n \geq 0 . \\
& v_{0}(x, y, t)=g(x, y), v_{n+1}(x, y, t)=L_{t}^{-1}\left[\frac{1}{s} L_{t}\left[\sum_{n=0}^{\infty} C_{n}+\sum_{n=0}^{\infty} D_{n}\right]\right], n \geq 0 .
\end{aligned}
$$

Note that the solution of (2.1), (2.2) can exhibit a shock phenomenon for finite $t$; we select $\mathrm{f}(\mathrm{x}, \mathrm{y})$ and $\mathrm{g}(\mathrm{x}, \mathrm{y})$ such that the shock occurs for a value of $t$ far from our region of interest. Let

$$
f(x, y)=g(x, y)=x+y
$$

Therefore from the recursive relation (2.9) and (2.10), we get

$$
u_{0}(x, y, t)=v_{0}(x, y, t)=x+y
$$

then $u_{1}(x, y, t), v_{1}(x, y, t)$ can be calculate as

$$
\begin{aligned}
u_{1}(x, y, t) & =L_{t}^{-1}\left[\frac{1}{s} L_{t}\left[A_{0}+B_{0}\right]\right] \\
& =L_{t}^{-1}\left[\frac{1}{s} L_{t}\left[u_{0} u_{0 x}+v_{0} u_{0 y}\right]\right] \\
& =L_{t}^{-1}\left[\frac{1}{s} L_{t}[(x+y)+(x+y)]\right] \\
& =2 t(x+y)
\end{aligned}
$$

Similarly,

$$
v_{1}(x, y, t)=L_{t}^{-1}\left[\frac{1}{s} L_{t}\left[C_{0}+D_{0}\right]\right]=L_{t}^{-1}\left[\frac{1}{s} L_{t}\left[u_{0} v_{0 x}+v_{0} v_{0 y}\right]\right]=2(x+y) t
$$


Also, $u_{2}(x, y, t)$ and $v_{2}(x, y, t)$ are calculated as

$$
\begin{aligned}
u_{2}(x, y, t) & =L_{t}^{-1}\left[\frac{1}{s} L_{t}\left[A_{1}+B_{1}\right]\right] \\
& =L_{t}^{-1}\left[\frac{1}{s} L_{t}\left[\left(u_{0} u_{1 x}+u_{1} u_{0 x}\right)+\left(v_{0} u_{1 y}+v_{1} u_{0 y}\right)\right]\right] \\
& =L_{t}^{-1}\left[\frac{1}{s} L_{t}[(2 t(x+y)+2 t(x+y))+(2 t(x+y)+2 t(x+y))]\right] \\
& =L_{t}^{-1}\left[\frac{1}{s} L_{t}[8 t(x+y)]\right] \\
& =2 t^{2}(x+y)
\end{aligned}
$$

Similarly,

$$
v_{2}(x, y, t)=4 t^{2}(x+y)
$$

Substitute all the values of $u_{0}, u_{1}, u_{2}, \cdots$ and $v_{0}, v_{1}, v_{2}, \cdots$ in the Equation (2.7), we get

$$
\begin{aligned}
& u(x, y, t)=(x+y)+2 t(x+y)+4 t^{2}(x+y)+\cdots \\
& v(x, y, t)=(x+y)+2 t(x+y)+4 t^{2}(x+y)+\cdots
\end{aligned}
$$

This implies,

$$
\begin{gathered}
u(x, y, t)=(x+y)\left[1+2 t+4 t^{2}+\cdots\right] \\
v(x, y, t)=(x+y)\left[1+2 t+4 t^{2}+\cdots\right] \\
u(x, y, t)=\frac{x+y}{1-2 t} \\
v(x, y, t)=\frac{x+y}{1-2 t}
\end{gathered}
$$

This is an exact solution of the given system of nonlinear partial differential Equations (2.1) and (2.2). We have verified this through the substitution, which is identical to the solution obtained by R. E. Bellman using the method of differential quadrature [15]. Let we change the initial conditions to

$$
f(x, y)=x^{2}, \quad g(x, y)=y
$$

From the recursive relation (2.9), (2.10) and above initial conditions, we get

$$
\begin{gathered}
u_{0}(x, y, t)=x^{2}, \quad v_{0}(x, y, t)=y \\
u_{1}(x, y, t)=L_{t}^{-1}\left[\frac{1}{s} L_{t}\left[A_{0}+B_{0}\right]\right]=2 x^{3} t
\end{gathered}
$$

Similarly,

$$
v_{1}(x, y, t)=L_{t}^{-1}\left[\frac{1}{s} L_{t}\left[C_{0}+D_{0}\right]\right]=y t
$$

Also, $u_{2}(x, y, t)$ and $v_{2}(x, y, t)$ are calculated as

$$
u_{2}(x, y, t)=L_{t}^{-1}\left[\frac{1}{s} L_{t}\left[A_{1}+B_{1}\right]\right]=5 x^{4} t
$$


Similarly,

$$
v_{2}(x, y, t)=y t^{2}, \quad u_{3}(x, y, t)=14 x^{5} t^{3}
$$

and so on. Substitute all the values of $u_{0}, u_{1}, u_{2}, \cdots$ and $v_{0}, v_{1}, v_{2}, \cdots$ in Equation (2.7), we get

$$
\begin{gathered}
u(x, y, t)=x^{2}\left(1+2 t x+5 t^{2} x^{2}+14 x^{2} t^{2}+\cdots\right) \\
v(x, y, t)=y\left(1+t+t^{2}+\cdots\right)=\frac{y}{1-t}
\end{gathered}
$$

(The shock occurs at $t=\frac{1}{4 x}$ ). This is an approximate solution of given system of equations.

\section{Conclusion}

From the examples above, we can clearly say that we can calculate $u(x, y, t)$ and $v(x, y, t)$ when explicitly solutions exist for given initial functions. More importantly, the methodology [1] [2] [3] does have potential application to the system of nonlinear partial differential equations and clearly in the case of stochastic parameters as well. The given system of equation has a unique solution for the given boundary conditions.

\section{Conflicts of Interest}

The authors declare no conflicts of interest regarding the publication of this paper.

\section{References}

[1] Handibag, S.S. and Karande, B.D. (2012) Application of Laplace Decomposition Method to Solve Linear and Nonlinear Heat Equation. International Journal of Applied Physics and Mathematics, 2, 369-371. https://doi.org/10.7763/IJAPM.2012.V2.137

[2] Handibag, S.S. and Karande, B.D. (2013) Existence the Solutions of Some Fifth-Order Kdv Equation by Laplace Decomposition Method. American Journal of Computational Mathematics, 3, 80-85. https://doi.org/10.4236/ajcm.2013.31013

[3] Khan, M. (2010) Application of Laplace Decomposition Method to Solve Nonlinear Coupled Partial Differential Equations. World Applied Sciences Journal, 9, 13-19.

[4] Liu, M.-H. and Guan, K.-Y. (2009) The Lie Group Integrality of the Fisher Type Travelling Wave Equation. Acta Mathematicae Sinica, 25, 305-320. https://doi.org/10.1007/s10255-007-7106-6

[5] Wazwaz, A.-M. (2008) The Hirota's Bilinear Method and the Tanh-Coth Method for Multiple Soliton Solutions of the Sawada-Kotera Kadomtsev-Petviashvili Equation. Applied Mathematics and Computation, 200, 160-166. https://doi.org/10.1016/j.amc.2007.11.001

[6] Borhanifar, A. and Kabir, M.M. (2009) New Periodic and Soliton Solutions by Application of Exp-Function Method for Nonlinear Evolution Equations. Journal of Computational and Applied Mathematics, 229, 158-167. https://doi.org/10.1016/j.cam.2008.10.052 
[7] Parkes, E.J. and Duffy, B.R. (1996) An Automated Tanh-Function Method for Finding Solitary Wave Solutions to Non-Linear Evolution Equations. Computer Physics Communications, 98, 288-300. https://doi.org/10.1016/0010-4655(96)00104-X

[8] Fan, E.G. and Hon, Y.C. (2003) Applications of Extended Tanh Method to Special Types of Nonlinear Equations. Applied Mathematics and Computation, 141, 351-358. https://doi.org/10.1016/S0096-3003(02)00260-6

[9] Tascan, F. and Bekir, A. (2009) Analytic Solutions of the (2+1)-Dimensional Nonlinear Evolution Equations Using the Sine-Cosine Method. Applied Mathematics and Computation, 215, 3134-3139. https://doi.org/10.1016/j.amc.2009.09.027

[10] Nadukandi, P., Oate, E. and Garcia, J. (2010) A High-Resolution Petrov-Galerkin Method for the 1D Convection-Diffusion-Reaction Problem. Computer Methods in Applied Mechanics and Engineering, 199, 525-546. https://doi.org/10.1016/j.cma.2009.10.009

[11] Zhou, J.K. (1986) Differential Transform and Its Application for Electrical Circuits. Huazhong University Press, Wuhan.

[12] Drazin, P.G. and Johnson, R.S. (1989) Solutions: An Introduction. Cambridge University Press, Cambridge. https://doi.org/10.1017/CBO9781139172059

[13] Liu, X.Q. and Bai, C.L. (2000) Exact Solutions of Some Fifth-Order Nonlinear Equations. Applied Mathematics: A Journal of Chinese Universities, 15, 28-32. https://doi.org/10.1007/s11766-000-0005-8

[14] Adomian, G. (1988) A Review of the Decomposition Method in Applied Mathematics. Journal of Mathematical Analysis and Applications, 135, 501-544. https://doi.org/10.1016/0022-247X(88)90170-9

[15] Bellman, R.E. and Adomian, G. (1985) Partial Differential Equations-New Methods for Their Treatment and Application. Reidel, Dordrecht. https://doi.org/10.1007/978-94-009-5209-6

[16] Adomian, G. (1983) Stochastic Systems. Academic Press, New York. 